Esta revista forma parte del acervo de la Biblioteca Jurídica Virtual del Instituto de Investigaciones Jurídicas de la UNAM www.juridicas.unam. $m x$

DOI: http://dx.doi.org/10.22201/iij.24487910e.2014.5.10057

http://biblio.juridicas.unam.mx

\title{
Candidaturas independientes. Experiencia Quintana Roo
}

\section{Jorge Manríquez Centeno* \\ Rafael Enrique Guzmán Acosta** Rocío Hernández Arévalo***}

* Consejero presidente del Consejo General del IEQROO.

* Consejero electoral y presidente de la Comisión de Partidos Políticos y Radiodifusión del IEQROO.

*** Directora de partidos políticos del IEQROO.

D. R. @ 2014. Universidad Nacional Autónoma de México-Instituto de Investigaciones Jurídicas. Revista Mexicana de Derecho Electoral, núm. 5, enero-junio de 2014, pp. 315-335, México, D. F. 
2012 fue el año en que la figura de candidaturas independientes se incluyó en la Constitución Política de los Estados Unidos Mexicanos, por medio de una reforma política. Siendo Quintana Roo, uno de los dos estados pioneros en aplicar las candidaturas independientes, al hacerlas efectivas a los ciudadanos en el proceso electoral desarrollado durante 2013.

Así, la reforma constitucional en materia política publicada el 9 de agosto de 2012 en el Diario Oficial de la Federación, dio como resultado la inserción de la figura de "candidaturas independientes", suprimiendo con ello el derecho exclusivo que los partidos políticos tenían para solicitar el registro de candidaturas; estableciendo de esa forma un sistema alterno para la postulación a cargos de elección popular por parte de los ciudadanos, garantizando su derecho a ser votados.

El decreto de reforma a través de sus artículos segundo y tercero transitorios, determinó que el H. Congreso de la Unión y las Legislaturas de los Estados debían realizar las adecuaciones necesarias a su legislación secundaria, derivadas de dicho Decreto, en un periodo no mayor a un año, contado a partir de su entrada en vigor.

En este marco, el gobernador constitucional del estado de Quintana Roo, presentó ante el Congreso del Estado, dos iniciativas de reforma en las que propuso la inserción de la figura de candidaturas independientes; la primera, el 7 de noviembre de 2012, planteando la modificación de los artículos 41 y 49 de la Constitución local (Decreto 170) y la segunda, el 30 de noviembre de 2012, para proponer la modificación de diversos ordenamientos legales en la materia, entre ellas la Ley Electoral de Quintana Roo al insertar un título sexto denominado "De las candidaturas independientes" (Decreto 199).

Dichos documentos fueron impugnados por los partidos Acción Nacional, de la Revolución Democrática y del Trabajo. El PAN expuso como fuentes de agravio los siguientes puntos:

- Violación al procedimiento legislativo, toda vez que para la creación del Decreto 170, no se cumplieron las etapas para llevar a cabo la reforma a la Constitución local, las cuales se encuentran reguladas por la propia Constitución del estado y la Ley Orgánica del Poder Legislativo del Estado.

- Que la legislatura del estado de Quintana Roo incluyó en su reforma constitucional que los candidatos independientes deberían 
observar las disposiciones que se establecían para las precampañas y campañas electorales, dando con esto libertad al legislador local para establecer reglas de participación del candidato ciudadano durante las precampañas electorales en las leyes secundarias, no obstante que la Constitución federal en ningún momento regulaba el derecho ciudadano a realizar precampañas, dado que dichos actos están relacionados con los procesos de selección de candidatos exclusivos de los partidos políticos

Por su parte, el PRD y el PT, en sus respectivas acciones de inconstitucionalidad argumentaron lo siguiente:

- Violaciones formales al procedimiento legislativo del decreto 170 atinente a la Constitución local.

- Insubsistencia del decreto 199, derivada de la determinación de inconstitucionalidad de las reformas a la Constitución local.

- Proceso de selección de candidatos independientes y su participación en las precampañas.

- Solicitud de registro por el principio de representación proporcional.

- Imprecisión al establecer límites en las erogaciones relacionadas con la obtención del respaldo ciudadano.

- Invasión a las facultades del Instituto Federal Electoral en lo atinente al acceso de los candidatos independientes a radio y televisión.

En su momento, la Suprema Corte de Justicia de la Nación resolvió la acción de inconstitucionalidad 67/2012 y sus acumulados declarando:

- La invalidez en su totalidad del Decreto 170, por el que se reformó la Constitución del estado de Quintana Roo, por vicios en el procedimiento legislativo para su aprobación.

- La permisión a los aspirantes sin partido para la realización de actos de promoción, siempre y cuando se sujetaran a la misma normatividad electoral que los candidatos con partido político.

- La autonomía de las legislaturas locales para configurar el marco normativo que debiera aplicarse a los candidatos sin partido político, de ahí que los ministros consideraron que las restricciones impuestas a los candidatos independientes contenidas en la Ley 
Electoral de Quintana Roo eran constitucionales, incluyendo el artículo en el cual se estipulaba que por cada distrito, demarcación o municipio sólo podría registrarse un candidato independiente o una sola planilla conformada por aspirantes sin partido.

- La constitucionalidad de los artículos que limitan las candidaturas independientes sólo a los cargos de mayoría relativa.

- La validación que la cantidad equivalente al $2 \%$ del padrón electoral, según la circunscripción territorial respectiva, fuera el número mínimo de manifestaciones de apoyo que un aspirante a candidato independiente debería obtener para poder registrarse como candidato independiente.

Una vez que la Suprema Corte de Justicia de la Nación hizo efectiva la aplicación de las candidaturas independientes, el Instituto Electoral de Quintana Roo (IEQROO) realizó diversas reuniones de trabajo con los representantes de los partidos políticos ante el Consejo General, a fin de integrar una propuesta de los Lineamientos, para regular las diversas etapas del procedimiento de las candidaturas independientes, que por primera vez fueron efectivas por los quintanarroenses en el proceso electoral local ordinario 2013, donde se eligieron a los veinticinco diputados locales y a los miembros de los ayuntamientos de los diez municipios en que se divide el territorio estatal.

Con fecha 19 de marzo del mismo año, los partidos políticos de la Revolución Democrática y Acción Nacional, presentaron el juicio de revisión constitucional SX-JDC-122/2013, ante la Sala Regional Xalapa del Tribunal Electoral del Poder Judicial de la Federación, por medio del cual impugnaron los lineamientos aprobados, y que finalmente fue resuelto por la Sala Superior de dicho Tribunal el 22 de abril, confirmando el contenido de los mencionados lineamientos.

Es importante mencionar que los lineamientos ya referidos contemplan una serie de etapas, que se describen en el título sexto de la Ley Electoral de Quintana Roo, denominado "De las candidaturas independientes", siendo éstas las siguientes: del solicitante, la obtención de respaldo ciudadano, la declaratoria de quienes tendrán el derecho a registrase como candidato independiente y el registro de candidato independiente.

De acuerdo con la disposición citada, la primera etapa del procedimiento de selección de candidatos independientes, inició con la aprobación por parte del Consejo General de la convocatoria y linea- 
Esta revista forma parte del acervo de la Biblioteca Jurídica Virtual del Instituto de Investigaciones Jurídicas de la UNAM

mientos el 16 de marzo de 2013 del proceso electoral local ordinario, mismos que fueron publicados posteriormente el 18 de marzo del presente año, y de acuerdo a la modalidad, los ciudadanos interesados presentaron sus solicitudes ante el órgano electoral local, en las siguientes fechas:

- Para miembros de los Ayuntamientos de mayoría relativa del 26 al 29 de marzo del año de la elección.

- Para diputados de mayoría relativa, del 5 al 8 de abril del año de la elección.

En dicho proceso electoral, el IEQROO recepcionó un total de ocho solicitudes en la modalidad de miembros de los Ayuntamientos y 18 en la modalidad de diputados, las cuales se muestran a continuación:

\section{Solicitudes}

\begin{tabular}{|c|c|}
\hline \multicolumn{2}{|c|}{ Planillas } \\
\hline Municipio & $\begin{array}{c}\text { Aspirante a candidato a presidente } \\
\text { municipal }\end{array}$ \\
\hline Othon P. Blanco & Luis Javier Ortiz Cardin \\
\hline Felipe Carrillo Puerto & Quintin Enrique Cervera Mendez \\
\hline Felipe Carrillo Puerto & Celestino Palomo Balam \\
\hline Cozumel & José Fernan Salazar Medina \\
\hline Benito Juárez & Gelmy Candelaria Villanueva Bojorquez \\
\hline Solidaridad & Juan Bautista Espinosa Palma \\
\hline Tulum & Gabriel Sifri Imenez \\
\hline Isla Mujeres & Juan María Basto Chacon \\
\hline
\end{tabular}

\begin{tabular}{|c|c|c|}
\hline \multicolumn{2}{|c|}{ Fórmulas } \\
\hline \multirow{2}{*}{ Distrito } & Aspirante a candidato a diputado & Cargo \\
\hline \multirow{2}{*}{ I } & Francisco Javier Torres Llanes & Propietario \\
\cline { 2 - 3 } & Luz Angélica Noverola Martin & Suplente \\
\hline \multirow{3}{*}{ II } & Delfina Yolanda Méndez Aguilar & Propietario \\
\cline { 2 - 3 } & Luis Villaseñor Madrigal & Suplente \\
\cline { 2 - 3 } & José Fernando Cristóbal Mendoza Espino & Propietario \\
\cline { 2 - 3 } & -------------- & Suplente \\
\hline \multirow{2}{*}{ III } & Manuel Manzo Méndez & Propietario \\
\cline { 2 - 3 } & Eliceo Nahuat Uc & Suplente \\
\hline \multirow{2}{*}{ IV } & David Gómez Tox & Propietario \\
\cline { 2 - 3 } & Uh Zazil Beh Puc Hernández & Suplente \\
\hline
\end{tabular}


Esta revista forma parte del acervo de la Biblioteca Jurídica Virtual del Instituto de Investigaciones Jurídicas de la UNAM

\begin{tabular}{|c|c|c|}
\hline \multicolumn{3}{|c|}{ Fórmulas } \\
\hline Distrito & Aspirante a candidato a diputado & Cargo \\
\hline \multirow{2}{*}{$\mathrm{V}$} & José Meléndez Torres & Propietario \\
\hline & Rosa María Pérez Reyna & Suplente \\
\hline \multirow{2}{*}{ VI } & Carlos Gerardo Ortiz Merlos & Propietario \\
\hline & Silvia Del Carmen Piña Gómez & Suplente \\
\hline \multirow{2}{*}{ VII } & Raúl Ramón González Manzano & Propietario \\
\hline & José Luis Chi Chiquil & Suplente \\
\hline \multirow{2}{*}{ VIII } & Niza Teresita Puerto Paredes & Propietario \\
\hline & Irene del Pilar Rivero Gómez & Suplente \\
\hline \multirow{2}{*}{ VIII } & Armando Pérez & Propietario \\
\hline & Luis Alberto Rodríguez Ríos & Suplente \\
\hline \multirow{2}{*}{ IX } & José Eduardo Galaviz Ibarra & Propietario \\
\hline & Adán Moisés Aranda Godoy & Suplente \\
\hline \multirow{2}{*}{$\mathrm{X}$} & Juan Lares Caamal & Propietario \\
\hline & Martha Noemi Velázquez Castellanos & Suplente \\
\hline \multirow{2}{*}{$\mathrm{XI}$} & Joed Saúl Pérez Calderón & Propietario \\
\hline & Sandra Evelyn Beristain Melgarejo & Suplente \\
\hline \multirow{2}{*}{ XII } & Fernando Javier España Vela & Propietario \\
\hline & Daniel Castro Rendón & Suplente \\
\hline \multirow{2}{*}{ XIII } & María Virginia Betanzos Moreno & Propietario \\
\hline & Xochitl Solís Poblete & Suplente \\
\hline \multirow{2}{*}{ XIV } & Ana Luisa Leal González & Propietario \\
\hline & Ignacio Arsénio Calderón Chan & Suplente \\
\hline \multirow{2}{*}{ XIV } & Luis Isidro Venegas Rodríguez & Propietario \\
\hline & Cruz Alberto Perez Pat & Suplente \\
\hline \multirow{2}{*}{$\mathrm{XV}$} & Gilberto Martínez Ríos & Propietario \\
\hline & Héctor Enrique Novelo Esquivel & Suplente \\
\hline
\end{tabular}

Culminado el periodo de presentación de las solicitudes, la Dirección de Partidos Políticos del IEQROO, contó con 24 horas para iniciar con el trabajo de revisión de los documentos de cada uno de los solicitantes para verificar el cumplimiento de los requisitos que la ley electoral prevé, siendo éstos los que a continuación se enlistan:

I. Copia certificada del acta de nacimiento;

II. Copia de la credencial para votar;

III. Original de la constancia de residencia y vecindad;

IV. El programa de trabajo que promoverán en caso de ser registrados como candidatos independientes, $y$

V. Manifestación escrita, bajo protesta de decir verdad, que cumple con los requisitos señalados por la Constitución particular para el cargo de 
elección popular de que se trate, así como lo dispuesto en la fracción I del artículo 32 de la Ley Electoral.

De dicha revisión se detectaron algunas inconsistencias, por tanto se otorgó a los solicitantes respectivos un plazo de 24 horas para solventarla, haciendo de su conocimiento que de no hacerlo se procedería al desechamiento de plano de la solicitud.

Seguidamente, el Consejo General del IEQROO, emitió un acuerdo definitivo por modalidad, mediante el cual se dieron a conocer los nombres de los ciudadanos que cumplieron con los requisitos legales previstos, obteniendo desde ese momento la denominación de aspirantes a candidatos independiente.

Cabe señalar que en esta etapa, se desecharon tres solicitudes, una en la modalidad de Ayuntamientos correspondiente al municipio de Isla Mujeres y dos en la modalidad de diputados correspondientes a los distritos electorales uninominales II y XIV, las causas que dieron origen al desechamiento fue el no haber cumplido con los requisitos legales que la normatividad electoral prevé, así como el haber presentado de manera extemporánea la solicitud, siendo el caso del aspirante a candidato independiente en la modalidad de diputado por el distrito XIV.

Obtenidos los resultados de esta etapa, el Consejo General procedió a emitir la declaratoria respectiva, este documento jurídico fue emitido cinco días después a la conclusión del plazo de recepción de manifestaciones ciudadanas, determinando así las candidaturas independientes que obtuvieron el derecho a registrase, siendo éstas las siguientes:

- 4 en la modalidad de Ayuntamientos de los municipios de Felipe Carrillo Puerto, Cozumel, Benito Juárez y Solidaridad.

- 10 en la modalidad de diputados correspondientes a los distritos electorales uninominales del VI al XV.

Cabe señalar, que todos los solicitantes pasaron a la segunda etapa denominada "obtención de respaldo ciudadano", en la cual, los aspirantes a candidatos independientes realizaron actos y actividades dirigidas a la ciudadanía en general, con la única finalidad de obtener el mayor número de respaldo de la ciudadanía, permitiéndose que el aspirante se identifique como precandidato independiente, abstenién- 
Esta revista forma parte del acervo de la Biblioteca Jurídica Virtual del Instituto de Investigaciones Jurídicas de la UNAM

dose en todo momento de solicitar el voto de los ciudadanos el día de la jornada electoral a favor de ellos.

Ahora bien, por cuanto a la cantidad de manifestaciones de respaldo ciudadano que los aspirantes a candidatos deben obtener, la ley electoral establece que sea de por lo menos el $2 \%$ de los ciudadanos registrados en el padrón electoral de la circunscripción estatal, municipal o distrital, según corresponda, con corte al 31 de diciembre del año previo a la elección. Cabe señalar que dicho porcentaje fue parte de los agravios de las acciones de inconstitucionalidad promovidas por el PRD y PT, sin embargo la SCJN validó el porcentaje bajo el argumento de la libre configuración legislativa de que gozan los congresos de los estados, para definir la reglamentación bajo la cual se regirá dicho procedimiento.

En los siguientes cuadros se muestra la cantidad mínima de manifestaciones de respaldo ciudadano que los aspirantes a candidatos independientes debían obtener de acuerdo a la modalidad en que participaron en el proceso electoral local ordinario 2013:

\begin{tabular}{|c|c|c|c|}
\hline Municipio & $\begin{array}{l}\text { Padrón electoral } \\
31-12-2012\end{array}$ & \multirow{12}{*}{$2 \%$} & $\begin{array}{l}\text { Número de manifestaciones } \\
\text { de respaldo ciudadano }\end{array}$ \\
\hline Othón P. Blanco & 158101 & & 3162 \\
\hline José María Morelos & 23249 & & 465 \\
\hline Felipe Carrillo Puerto & 46714 & & 934 \\
\hline Cozumel & 61263 & & 1225 \\
\hline Solidaridad & 127560 & & 2551 \\
\hline Benito Juárez & 475846 & & 9517 \\
\hline Isla Mujeres & 16190 & & 324 \\
\hline Lázaro Cárdenas & 18069 & & 361 \\
\hline Tulum & 22186 & & 444 \\
\hline Bacalar & 25843 & & 517 \\
\hline Totales & 975021 & & 19500 \\
\hline
\end{tabular}


Esta revista forma parte del acervo de la Biblioteca Jurídica Virtual del Instituto de Investigaciones Jurídicas de la UNAM www.juridicas.unam.mx

DOI: http://dx.doi.org/10.22201/iij.24487910e.2014.5.10057

http://biblio.juridicas.unam.mx

\begin{tabular}{|c|c|c|c|}
\hline Distrito & $\begin{array}{l}\text { Padrón electoral } \\
\text { 31-12-2012 }\end{array}$ & \multirow{17}{*}{$2 \%$} & $\begin{array}{c}\text { Número de manifestaciones de } \\
\text { respaldo ciudadano }\end{array}$ \\
\hline I & 58684 & & 1174 \\
\hline II & 71496 & & 1430 \\
\hline III & 53764 & & 1075 \\
\hline IV & 69963 & & 1399 \\
\hline $\mathrm{V}$ & 79268 & & 1585 \\
\hline VI & 61263 & & 1225 \\
\hline VII & 70478 & & 1410 \\
\hline VIII & 57498 & & 1150 \\
\hline IX & 77518 & & 1550 \\
\hline $\mathrm{X}$ & 64499 & & 1290 \\
\hline $\mathrm{XI}$ & 77654 & & 1553 \\
\hline XII & 61292 & & 1226 \\
\hline XIII & 58818 & & 1176 \\
\hline XIV & 54614 & & 1092 \\
\hline $\mathrm{XV}$ & 58212 & & 1164 \\
\hline Totales & 975021 & & 19500 \\
\hline
\end{tabular}

Los plazos legales que contempla la normatividad local para la obtención del respaldo ciudadano son las siguientes:

- Para miembros de los Ayuntamientos del 4 al 18 de abril del año de la elección.

- Para diputados del 14 al 25 de abril del año de la elección.

Para tales efectos, el IEQROO instaló quince módulos de recepción de manifestaciones de respaldo ciudadano durante los periodos arriba señalados, para que los ciudadanos interesados en emitir su apoyo por algún candidato independiente, acudieran personalmente ante el funcionario electoral y previa identificación con su credencial para votar vigente, se le proporcionaba el formato de manifestación de respaldo ciudadano para su llenado de puño y letra.

Cabe señalar que para la recepción de las manifestaciones de apoyo por las modalidades antes referidas, se procedió conforme a las siguientes reglas:

I) Las manifestaciones de respaldo se requisitaron en el momento de su entrega en el formato correspondiente que para tal efecto 
aprobó el Consejo General, mismo que fue firmado o huellado por el ciudadano directamente interesado.

II) La recepción de las manifestaciones se hizo ante la presencia de los funcionarios electorales designados y de los representantes que, en su caso, designaron los partidos políticos o coaliciones y que los propios aspirantes acreditaron.

III) Las manifestaciones de respaldo para aspirantes a candidatos a diputados se presentaron en la sede del Consejo Distrital correspondiente a la demarcación por la que el aspirante a candidato independiente estuviera compitiendo, y exclusivamente por ciudadanos con domicilio en ese ámbito territorial.

IV) Las manifestaciones de apoyo para aspirantes a los Ayuntamientos se presentaron ante la sede del Consejo Municipal, Distrital o Distritales correspondientes a la demarcación por la que el aspirante a candidato estuviera compitiendo, y exclusivamente por los ciudadanos con domicilio en el municipio de que se trate.

Es de señalarse que durante el proceso de obtención de respaldo ciudadano, se recibieron ante la oficialía de partes del IEQROO, dos escritos de los aspirantes a candidatos en la modalidad de diputados por los distritos IV y XIV, mediante los cuales se solicitó al Consejo General la instalación de módulos de recepción adicionales a los ya determinados para tales efectos.

Por lo que el Consejo General aprobó mediante acuerdos que no resultaban procedentes las solicitudes antes referidas, en razón de que previo a la etapa de obtención de respaldo ciudadano, el Consejo General aprobó los Lineamientos y la Convocatoria donde se establecieron las sedes de los módulos de recepción de las manifestaciones de respaldo ciudadano, lo anterior, toda vez que el artículo 132, fracción IV de la Ley Electoral de Quintana Roo, establece que las manifestaciones de respaldo de aspirantes a candidatos independientes a diputados serán presentados en la sede del Consejo Distrital que corresponda a la demarcación por la que se pretenda competir, y exclusivamente por ciudadanos con domicilio en ese ámbito territorial; entendiéndose como la sede a la cabecera distrital en la cual residirá dicho órgano desconcentrado.

Aunado a lo anterior es de señalarse que al no haber sido materia de impugnación los documentos antes referidos, estos quedaron definiti- 
vos y firmes, en lo que respecta a los domicilios en los cuales se llevó a cabo la recepción de la manifestación de respaldo ciudadano.

Una vez que concluyeron los periodos de recepción, los servidores electorales designados para la atención de cada módulo instalado, remitieron las manifestaciones recepcionadas a la Dirección de Partidos Políticos del IEQROO, para corroborar el número de manifestaciones que cada aspirante obtuvo, así como también para su respectiva clasificación de acuerdo a los supuestos establecidos en el artículo 133 de la ley electoral, que las precisa como nulas o válidas conforme a lo siguiente:

I) Cuando se haya presentado, por la misma persona, más de una manifestación a favor del mismo aspirante, debiendo prevalecer únicamente la primera que haya sido registrada.

II) Cuando se hayan expedido por la misma persona a dos o más aspirantes al mismo cargo de elección popular.

III) Cuando carezcan de la firma o, en su caso, huella o datos de identificación en el formato previsto para tal efecto, o bien, cuando tales datos no sean localizados en el padrón electoral.

IV) Cuando los ciudadanos que las expidan hayan sido dados de baja del padrón electoral por encontrarse en alguno de los supuestos señalados en la legislación aplicable.

V) Cuando los ciudadanos que las expidan no correspondan al ámbito estatal, distrital o municipal por el que el aspirante pretenda competir.

Es de señalarse que la normatividad electoral únicamente permite el registro de un candidato independiente por modalidad; por lo que cuando uno de ellos haya obtenido por los menos el $2 \%$ de manifestaciones de respaldo ciudadano, o en su caso, de resultar que más de uno obtenga el porcentaje, se le otorgará tal derecho al que haya obtenido el mayor número de respaldos.

Obtenidos los resultados de esta etapa, el Consejo General del IEQROO procedió a integrar la Declaratoria respectiva, documento jurídico que se emitió cinco días después de la conclusión del plazo de recepción de manifestaciones de respaldo ciudadano, determinando así a los aspirantes a candidatos independientes que continúan participando en el procedimiento; en el caso que nos ocupa, las candidaturas que continuaron fueron las siguientes: 
Esta revista forma parte del acervo de la Biblioteca Jurídica Virtual del Instituto de Investigaciones Jurídicas de la UNAM www.juridicas.unam.mx

DOI: http://dx.doi.org/10.22201/iij.24487910e.2014.5.10057

http://biblio.juridicas.unam.mx

- 4 en la modalidad de Ayuntamientos, correspondientes a los municipios de Felipe Carrillo Puerto, Cozumel, Benito Juárez y Solidaridad; correspondiente a los ciudadanos siguientes:

\begin{tabular}{|c|c|c|}
\hline Municipio & $\begin{array}{c}\text { Aspirante a candidato independiente } \\
\text { a presidente municipal }\end{array}$ & Emblema \\
\hline Felipe Carrillo Puerto & Celestino Palomo Balam & \\
\hline Cozumel & José Fernán Salazar Medina & \\
\hline Benito Juárez & $\begin{array}{c}\text { Gelmy Candelaria Villanueva } \\
\text { Bojórquez }\end{array}$ & \\
\hline Solidaridad & Juan Bautista Espinosa Palma & $\begin{array}{c}\text { Ciudadanos Unidos } \\
\text { por Solidaridad }\end{array}$ \\
\hline
\end{tabular}

- 10 en la modalidad de diputados, correspondientes a los distritos electorales uninominales del VI al XV, correspondiente a los ciudadanos siguientes:

\begin{tabular}{|c|c|c|c|}
\hline \multirow{2}{*}{ Distrito } & Nombre & Calidad \\
\hline \multirow{3}{*}{ VI } & Carlos Gerardo Ortiz Merlos & Propietario \\
\cline { 2 - 4 } & Silvia del Carmen Piña Gómez & Suplente \\
\hline \multirow{2}{*}{ VII } & Raúl Ramón González Manzano & Propietario \\
\cline { 2 - 4 } & José Luis Chi Chiquil & Suplente \\
\hline \multirow{3}{*}{ VIII } & Niza Teresita Puerto Paredes & Propietario \\
\cline { 2 - 4 } & Irene Del Pilar Rivero Gómez & Suplente \\
\hline \multirow{3}{*}{ IX } & José Eduardo Galaviz Ibarra & Propietario \\
\cline { 2 - 4 } & Adán Moisés Aranda Godoy & Suplente \\
\hline \multirow{2}{*}{$\mathrm{X}$} & Juan Lares Caamal & Propietario \\
\cline { 2 - 4 } & Martha Velázquez Castellanos & Suplente \\
\hline
\end{tabular}


Esta revista forma parte del acervo de la Biblioteca Jurídica Virtual del Instituto de Investigaciones Jurídicas de la UNAM

\begin{tabular}{|c|c|c|c|}
\hline Distrito & Nombre & Calidad & EMBLEMA \\
\hline \multirow{2}{*}{$\mathrm{XI}$} & Joed Saúl Pérez Calderón & Propietario & \\
\hline & Sandra Evelyn Beristain Melgarejo & Suplente & \\
\hline \multirow{2}{*}{ XII } & Fernando Javier España Vela & Propietario & NORTE \\
\hline & Daniel Castro Rendón & Suplente & \\
\hline \multirow{2}{*}{ XIII } & María Virginia Betanzos Moreno & Propietario & \\
\hline & Xochitl Solís Poblete & Suplente & \\
\hline \multirow{2}{*}{ XIV } & Ana Luisa Leal González & Propietario & \\
\hline & Ignacio Arsénio Calderón Chan & Suplente & \\
\hline \multirow{2}{*}{$\mathrm{XV}$} & Gilberto Martínez Ríos & Propietario & \\
\hline & Héctor Enrique Novelo Esquivel & Suplente & \\
\hline
\end{tabular}

Es importante señalar que para llevar a cabo las actividades de obtención de respaldo ciudadano, únicamente pudieron ser sufragadas con financiamiento privado, considerando que dicho importe recibido durante esta etapa más lo que pudiera recibir durante la campaña electoral no podría exceder, en su conjunto del $10 \%$ del último tope fijado para la elección de gobernador y que las aportaciones personales de los candidatos independientes están incluidas dentro del tope señalado.

Asimismo la ley electoral prevé que el otorgamiento del financiamiento público es hasta lograr su registro como candidato independiente, considerando que, en la modalidad de Ayuntamiento, tendrían derecho a recibir financiamiento público considerando el monto que corresponda como si se tratara de un partido de nueva creación y cada uno en la proporción al número de electores inscritos en la demarcación por la que compitan, de igual forma para diputados por mayoría relativa.

De igual forma, se prevé que las personas físicas o morales mexicanas con residencia en el país pueden realizar aportaciones y donativos, con la salvedad de que no se traten de partidos políticos o de alguno de los tres poderes del estado; dependencias u organismos de la administración pública; organismos internacionales; ministros de culto 
o agrupaciones de cualquier culto religioso; así como las demás que se contemplan en el artículo 92 de la Ley Electoral.

En el caso de los Topes de gastos de los candidatos independientes, la normatividad electoral establece que serán los mismos montos que se determinen para los partidos políticos.

A continuación se desglosan los montos a los que ascendió el financiamiento público y privado, así como los topes de gastos de campaña de las candidaturas independientes que participaron en el proceso electoral ordinario 2013. 
Esta revista forma parte del acervo de la Biblioteca Jurídica Virtual del Instituto de Investigaciones Jurídicas de la UNAM www.juridicas.unam. $\mathrm{mx}$

DOI: http://dx.doi.org/10.22201/iij.24487910e.2014.5.10057

http://biblio.juridicas.unam.mx

0
0
0
0
0
0
0
0
0
0
0
0
0
0
0
0
0
0
0
0
0
0
0
0
0
0
0
0
0
0

\begin{tabular}{|c|c|c|c|c|c|c|c|c|c|c|c|c|c|}
\hline \multicolumn{3}{|c|}{ 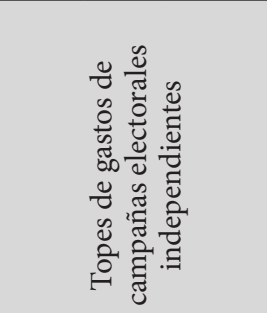 } & 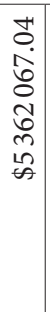 & 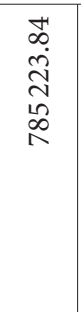 & 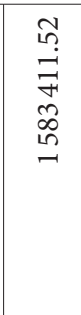 & 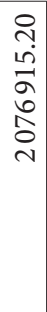 & 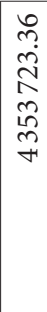 & 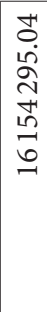 & 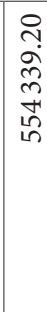 & 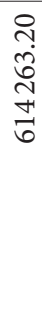 & 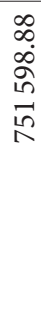 & 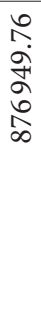 & \\
\hline \multicolumn{3}{|c|}{ 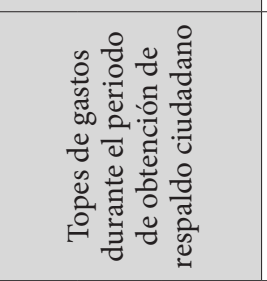 } & 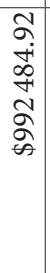 & $\begin{array}{l}\circ \\
\stackrel{1}{0} \\
\stackrel{1}{1} \\
\infty \\
\text { I } \\
\end{array}$ & $\begin{array}{l}\stackrel{n}{n} \\
= \\
\tilde{n} \\
D \\
\stackrel{n}{n}\end{array}$ & $\begin{array}{l}\hat{N} \\
\hat{N} \\
0 \\
\bar{n} \\
\end{array}$ & $\begin{array}{l}\infty \\
-1 \\
8 \\
0 \\
0 \\
0 \\
0 \\
1 n\end{array}$ & 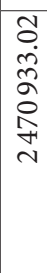 & 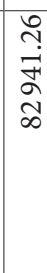 & 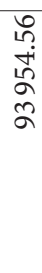 & 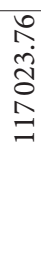 & $\begin{array}{l}\text { No } \\
\infty \\
\stackrel{0}{0} \\
\stackrel{+}{+} \\
m \\
\sim\end{array}$ & \\
\hline \multicolumn{3}{|c|}{ 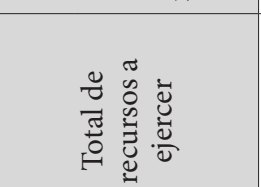 } & 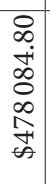 & $\begin{array}{l}\text { N̦ } \\
\text { ปิ } \\
\stackrel{0}{0}\end{array}$ & $\begin{array}{l}\text { I } \\
\stackrel{\Lambda}{1} \\
\text { In } \\
\exists \\
\exists\end{array}$ & 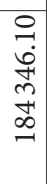 & $\begin{array}{l}\tilde{b} \\
\dot{0} \\
\infty \\
\infty \\
\infty \\
\infty \\
m\end{array}$ & 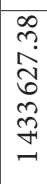 & $\begin{array}{l}\infty \\
10 \\
100 \\
2 \\
\infty \\
\infty \\
+1\end{array}$ & तू & 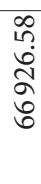 & $\begin{array}{l}\text { O+ } \\
\dot{0} \\
\infty \\
N \\
N\end{array}$ & \\
\hline 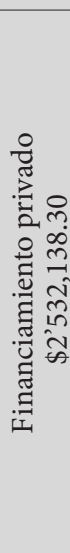 & 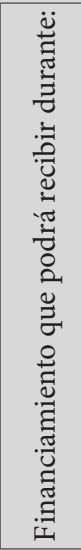 & 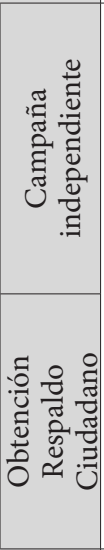 & 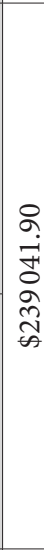 & 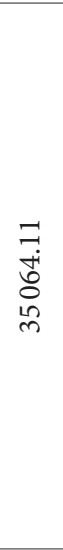 & 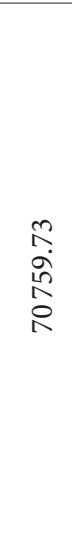 & 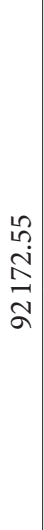 & $\begin{array}{l}\vec{n} \\
\dot{z} \\
2 \\
\vdots \\
\vdots\end{array}$ & $\begin{array}{l}2 \\
\vec{m} \\
\infty \\
\infty \\
0 \\
1\end{array}$ & 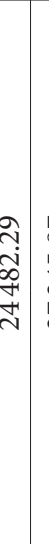 & \multicolumn{2}{|c|}{ 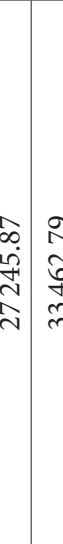 } & 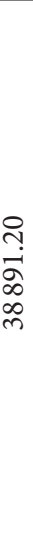 & $\begin{array}{l}2 \\
+ \\
\infty \\
\infty \\
0 \\
+\end{array}$ \\
\hline \multicolumn{3}{|c|}{ 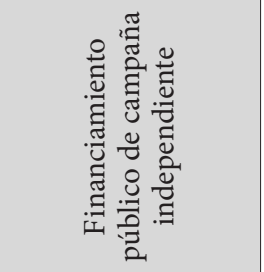 } & 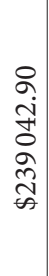 & 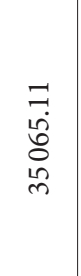 & 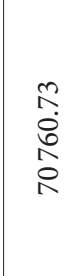 & 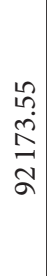 & 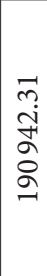 & 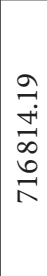 & \begin{tabular}{l} 
సे \\
ஸे \\
$\infty$ \\
+ \\
\multirow{+}{*}{}
\end{tabular} & $\begin{array}{l}\infty \\
\infty \\
\dot{0} \\
\stackrel{+}{N} \\
\text { N }\end{array}$ & $\begin{array}{l}\hat{N} \\
\text { ñ } \\
\text { +े } \\
m \\
m\end{array}$ & 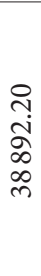 & \\
\hline \multicolumn{3}{|c|}{ 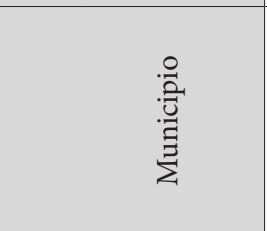 } & 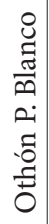 & 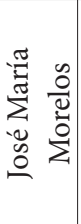 & 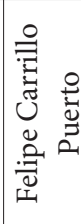 & 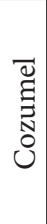 & 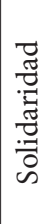 & 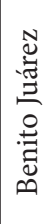 & 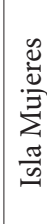 & 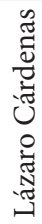 & $\underset{\Xi}{\Xi}$ & 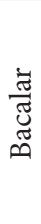 & \\
\hline
\end{tabular}




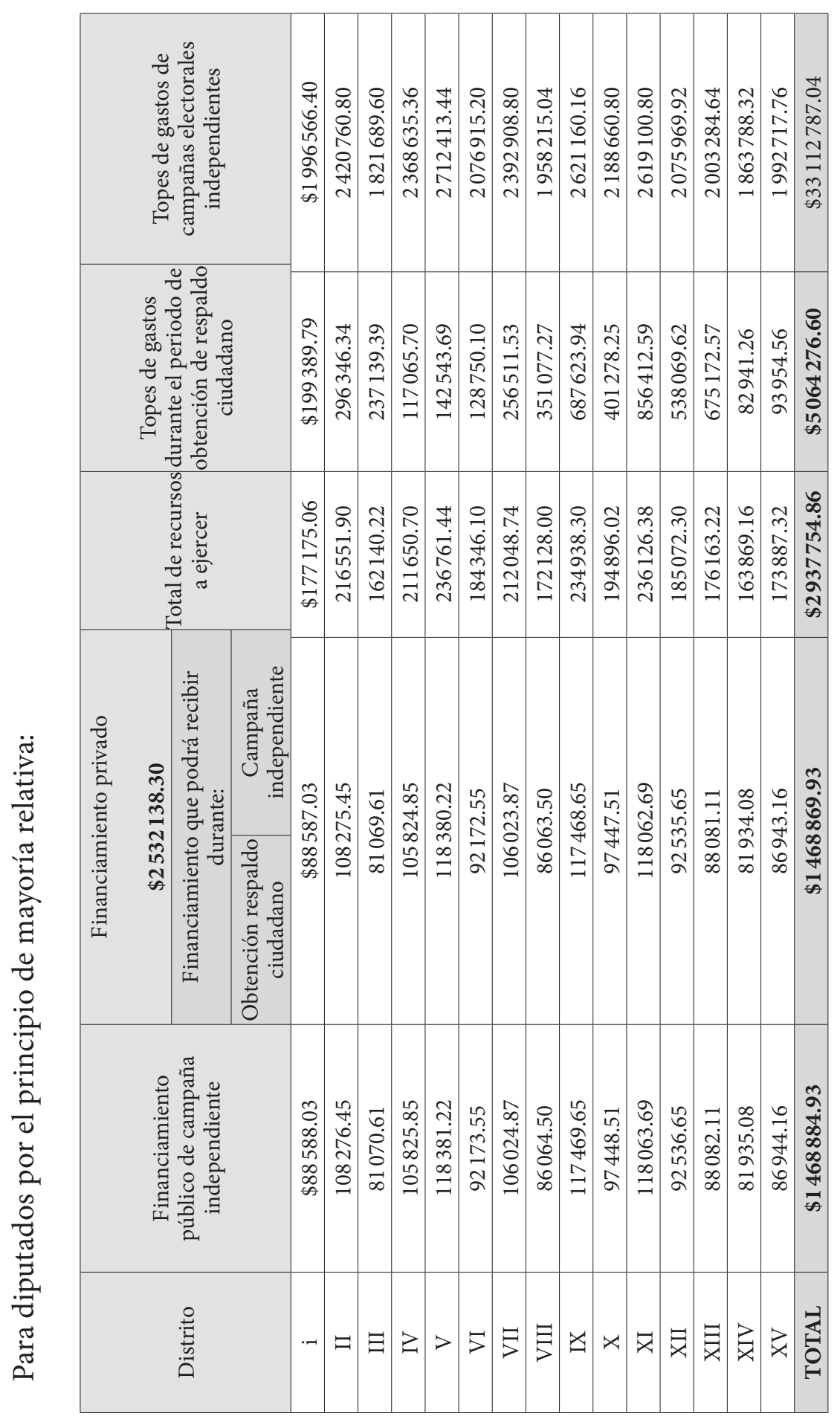


Esta revista forma parte del acervo de la Biblioteca Jurídica Virtual del Instituto de Investigaciones Jurídicas de la UNAM

En este mismo tenor, una vez que los aspirantes obtienen el derecho a registrarse como candidatos independientes, deben presentar ante la Dirección de Partidos Políticos, dentro de los dos días posteriores a la emisión de la Declaratoria, un informe de comprobación de gastos de la obtención del respaldo ciudadano, anexando la documentación soporte y comprobatoria de sus gastos, en los plazos que a continuación se muestran:

\begin{tabular}{|c|c|c|c|}
\hline $\begin{array}{l}\text { Entrega de informes } \\
\text { (2 días posteriores } \\
\text { a la emisión de la } \\
\text { declaratoria) }\end{array}$ & $\begin{array}{l}\text { Fecha límite para } \\
\text { emisión oficios de } \\
\text { observaciones }\end{array}$ & $\begin{array}{l}\text { Recepción de } \\
\text { aclaraciones }\end{array}$ & $\begin{array}{l}\text { Fecha límite para } \\
\text { elaboración de } \\
\text { dictámenes }\end{array}$ \\
\hline $\begin{array}{l}\text { Miembros de los } \\
\text { Ayuntamientos }\end{array}$ & $\begin{array}{l}8 \text { días después de } \\
\text { recibidos }\end{array}$ & $\begin{array}{c}3 \text { días siguientes a la } \\
\text { notificación }\end{array}$ & 7 días \\
\hline 25 de abril & & & (12 de mayo de 2013) \\
\hline $\begin{array}{c}\text { Diputados por } \\
\text { principio de mayoría } \\
\text { relativa }\end{array}$ & $\begin{array}{l}6 \text { días después de } \\
\text { recibidos }\end{array}$ & $\begin{array}{c}3 \text { días siguientes a la } \\
\text { notificación }\end{array}$ & 7 días \\
\hline 2 de mayo & (7 de mayo 2013) & (10 de mayo de 2013) & (17 de mayo de 2013) \\
\hline
\end{tabular}

Concluida la revisión de los gastos que fueron erogados durante la etapa en cuestión, el Consejo General emite un día antes del inicio del plazo de registro de candidaturas según la modalidad, el Dictamen en el cual se verificó que los gastos erogados por los aspirantes a candidatos independientes se encontraran dentro del tope y los montos máximos de aportación permitido para el proceso electoral local ordinario 2013.

Es de resaltarse, que, al finalizar la etapa de obtención de respaldo ciudadano y una vez emitida la declaratoria de quienes tendrían derecho a registrarse como candidatos independientes, el proceso de registro de los mismos coincide con los de los candidatos postulados por los partidos políticos.

En esta etapa de registro, los aún aspirantes a candidatos independientes presentan su solicitud ante la autoridad electoral, anexando la ratificación de su programa de trabajo y el dictamen de la licitud de los gastos; asimismo proporcionan el nombre de su representante y del responsable de la administración de los recursos financieros y la presentación de los informes de campaña, señalan los colores y en su caso de los emblemas a utilizar en su propaganda electoral (los cuales 
Esta revista forma parte del acervo de la Biblioteca Jurídica Virtual del Instituto de Investigaciones Jurídicas de la UNAM

no podían ser iguales o semejantes a los utilizados por los partidos políticos); las fechas en las que se presentaron las solicitudes de registro son las siguientes:

- Para miembros de los Ayuntamientos, el 8 de mayo de 2013 de la elección, ante el Consejo General.

- Para diputados por el principio de mayoría relativa; el 14 de mayo de 2013, ante el Consejo General.

Las observaciones que se derivaron de la revisión de la documentación antes referida, fueron notificados a los interesados, a través de la Dirección de Partidos Políticos, otorgándole un término de 48 horas para subsanarlas; una vez agotado este término, el Consejo General sesionó en las fechas establecidas por la normatividad electoral para registrar a las candidaturas independientes en la modalidad correspondiente, cabe señalar que todos los ciudadanos solicitantes a los que se les otorgó la declaratoria respectiva, fueron registrados como candidatos independientes.

Una vez que los ciudadanos obtuvieron su registro como candidatos independientes, el IEQROO procedió a dar aviso al Instituto Federal Electoral (IFE) en un plazo no mayor a 72 horas para que los candidatos independientes estuvieran en aptitud de tener acceso a tiempos de radio y televisión.

El IFE realizó una propuesta de distribución, tomando en cuenta el número de candidatos independientes registrados para cada cargo de elección popular para el proceso electoral local ordinario 2013, del cual se obtuvo el siguiente resultado: 
Esta revista forma parte del acervo de la Biblioteca Jurídica Virtual del Instituto de Investigaciones Jurídicas de la UNAM www.juridicas.unam.mx

DOI: http://dx.doi.org/10.22201/iij.24487910e.2014.5.10057

http://biblio.juridicas.unam.mx

\begin{tabular}{|c|c|c|c|c|c|c|c|c|c|c|c|}
\hline & & 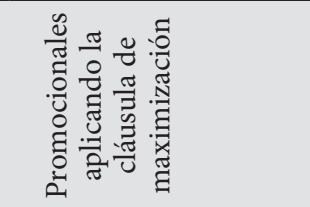 & 유 & $\overrightarrow{\widehat{\lambda}}$ & $\overrightarrow{\sqrt{2}}$ & શે & $\bar{\beth}$ & $\stackrel{0}{\sim}$ & $\stackrel{\infty}{\sim}$ & $\stackrel{R}{R}$ & $\begin{array}{l}0 \\
\stackrel{0}{\circ} \\
\infty\end{array}$ \\
\hline 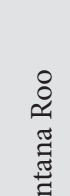 & & 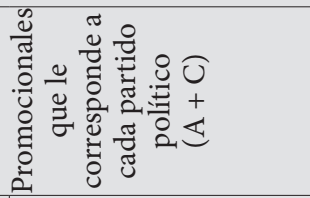 & 윰 & $\overrightarrow{\widehat{\lambda}}$ & $\overrightarrow{\mathfrak{n}}$ & $\stackrel{\text { }}{=}$ & $\overrightarrow{\vec{v}}$ & $\underset{\sim}{\sim}$ & $\stackrel{\infty}{\underset{J}{(-1}}$ & $\stackrel{P}{R}$ & 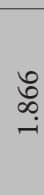 \\
\hline 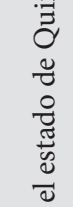 & 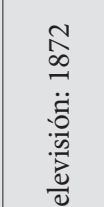 & 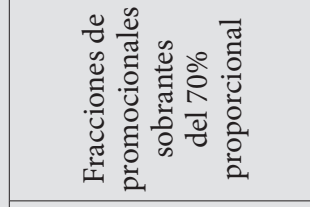 & $\begin{array}{l}\text { 우 } \\
\text { b } \\
\text { o }\end{array}$ & \begin{tabular}{l}
0 \\
\multirow{N}{N}{} \\
$\hat{n}$ \\
0
\end{tabular} & $\begin{array}{l}\text { R } \\
\hat{a} \\
0 \\
0\end{array}$ & 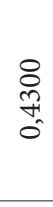 & \begin{tabular}{l}
$\infty$ \\
\multirow{2}{N}{} \\
0
\end{tabular} & $\begin{array}{l}\stackrel{8}{N} \\
\text { Nิ }\end{array}$ & $\begin{array}{l}\text { \& } \\
\text { Цू } \\
\text { o }\end{array}$ & $\begin{array}{l}8 \\
8 \\
8 \\
0\end{array}$ & $\stackrel{8}{\circ}$ \\
\hline 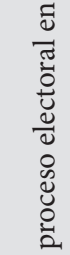 & 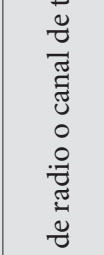 & 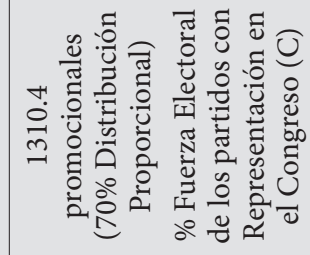 & ஓ্ণ & $\overrightarrow{\widetilde{N}}$ & $\underset{\sim}{-\infty}$ & જิ & $\underset{\Xi}{\exists}$ & $\stackrel{l}{+1}$ & $\stackrel{\infty}{\infty}$ & 0 & 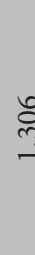 \\
\hline 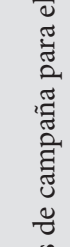 & 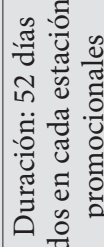 & 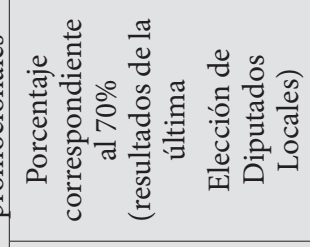 & $\begin{array}{c}8 \\
\text { 秀 } \\
\text { 命 }\end{array}$ & $\begin{array}{l}8 \\
\stackrel{\sigma}{a} \\
\sigma \\
-\end{array}$ & $\begin{array}{l}8 \\
\stackrel{1}{\infty} \\
\infty \\
\stackrel{n}{2}\end{array}$ & $\begin{array}{l}8 \\
8 \\
m \\
\text { in }\end{array}$ & $\begin{array}{l}8 \\
\infty \\
\hat{0} \\
0\end{array}$ & $\begin{array}{l}\stackrel{8}{0} \\
= \\
=\end{array}$ & $\begin{array}{l}8 \\
8 \\
\text { in } \\
f\end{array}$ & $\begin{array}{l}8 \\
8 \\
0 \\
0\end{array}$ & 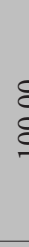 \\
\hline 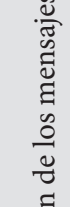 & 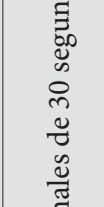 & 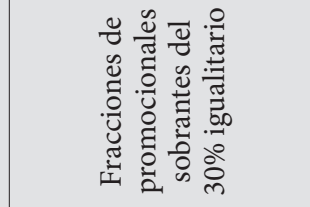 & $\begin{array}{l}\stackrel{\circ}{1} \\
\stackrel{0}{0}\end{array}$ & 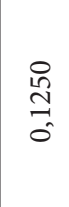 & $\stackrel{\stackrel{\leftrightarrow}{n}}{\stackrel{1}{0}}$ & $\begin{array}{l}\stackrel{0}{n} \\
\stackrel{0}{1}\end{array}$ & 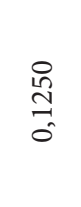 & $\begin{array}{l}0 \\
\stackrel{1}{1} \\
0 \\
0\end{array}$ & $\stackrel{\substack{10\\
}}{0}$ & $\begin{array}{l}\stackrel{0}{10} \\
\stackrel{1}{6} \\
0\end{array}$ & $\varepsilon$ \\
\hline 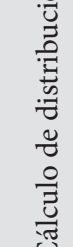 & 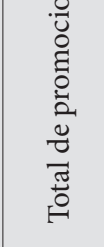 & 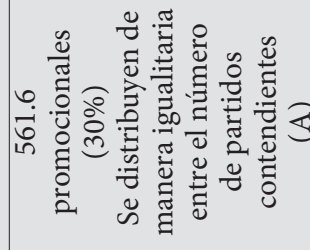 & $\stackrel{R}{ }$ & $\stackrel{R}{R}$ & $R$ & $\stackrel{R}{ }$ & $R$ & $\stackrel{R}{R}$ & $\stackrel{R}{R}$ & $R$ & i̊ \\
\hline & & 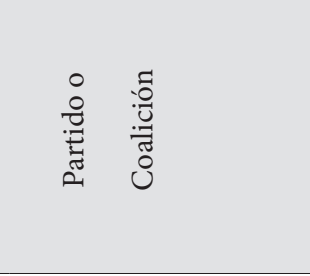 & 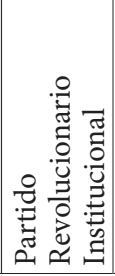 & 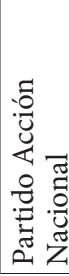 & 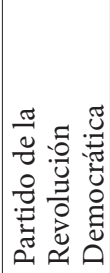 & 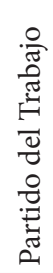 & 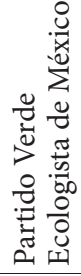 & 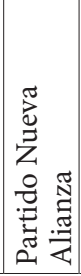 & 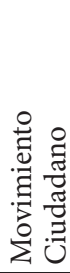 & 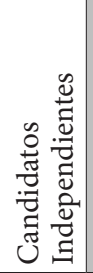 & 昵 \\
\hline
\end{tabular}


Con el registro de los candidatos independientes se dio inicio al periodo de campañas, conjuntamente con los candidatos de los partidos políticos en donde éstos dieron a conocer a la ciudadanía quintanarroense sus programas de acción con la finalidad de obtener el voto el día de la jornada electoral.

Así los estados que realizaron adecuaciones a sus Constituciones locales y normatividad electoral en términos de candidaturas independientes hasta septiembre de 2013 son Aguascalientes, Baja California, Baja California Sur, Chiapas, Coahuila, Colima, Durango, Estado de México, Guerrero, Hidalgo, Jalisco, Michoacán, Nayarit, Nuevo León, Puebla, Querétaro, San Luis Potosí, Sinaloa, Tabasco, Tamaulipas y Tlaxcala.

En conclusión, la inserción de la figura de candidaturas independientes en la normatividad electoral del Estado de Quintana Roo, se tradujo en el cumplimiento al objetivo de la reforma federal en materia política, contribuyendo así al ejercicio pleno del derecho al voto pasivo de los ciudadanos, que sin ser respaldados por un partido político, pudieron ser votados a los distintos cargos de elección popular de la entidad. 
Esta revista forma parte del acervo de la Biblioteca Jurídica Virtual del Instituto de Investigaciones Jurídicas de la UNAM www.juridicas.unam.mx DOI: http://dx.doi.org/10.22201/iij.24487910e.2014.5.10057 http://biblio.juridicas.unam.mx 